\title{
Differential expression of TYRP1 in adult human retinal pigment epithelium and uveal melanoma cells
}

\author{
CHUN QIU ${ }^{1,2^{*}}$, PENG LI $^{1,2^{*}}$, JIANJUN BI $^{2}$, QING WU ${ }^{2}$, LINNA LU $^{2}$, \\ GUANXIANG QIAN $^{2}$, RENBING JIA ${ }^{2}$ and RONG JIA ${ }^{1}$ \\ ${ }^{1}$ School of Life Science, Anhui University, Hefei, Anhui 230601; ${ }^{2}$ Department of Ophthalmology, Ninth People's Hospital, \\ Shanghai Jiao Tong University, School of Medicine, Shanghai 200011, P.R. China
}

Received November 1, 2014; Accepted November 16, 2015

DOI: $10.3892 / \mathrm{ol} .2016 .4280$

\begin{abstract}
Uveal melanoma (UM) is the most frequently occurring primary intraocular malignancy in adults. Tyrosinase (TYR) is a copper-containing enzyme and a type I membrane protein that is involved in the generation of melanin, the main pigment in vertebrates. TYR-related protein 1 (TYRP1) is regarded to have a crucial role in the immunotherapy of melanoma. As biomarkers, the TYR-related proteins, TYRP1 and TYRP2, exhibit specific expression in melanocytes, while also contributing to melanin synthesis within melanosomes. In the present study, the differential expression of TYRP1 was investigated at the mRNA, protein and morphological levels in four human UM cell lines (SP6.5, OM431, OCM1 and OCM290) and the human retinal pigment epithelium (RPE) cell line, using polymerase chain reaction, western blotting, immunocytochemistry and immunofluorescence staining. It was found that SP6.5 cells expressed the highest level of TYRP1, in comparison to SP6.5 OCM1 and OM431 cells, which produced less TYRP1, and OCM290 cells, which produced almost no TYRP1. No TYRP1 protein expression was identified in the RPE cell line. These findings indicate the potential use of TYRP1 in the development of therapy for UM.
\end{abstract}

\section{Introduction}

As the most common primary intraocular malignant tumor in adults, uveal melanoma (UM) has an incidence of 7 cases in

Correspondence to: Professor Rong Jia, School of Life Science, Anhui University, 111 Jiulong Road, Hefei, Anhui 230601, P.R. China E-mail: jiaronganhui@outlook.com

Dr Renbing Jia, Department of Ophthalmology, Ninth People's Hospital, Shanghai Jiao Tong University, School of Medicine, 639 Zhizaoju Road, Shanghai 200011, P.R. China

E-mail: jrb19760517@hotmail.com

*Contributed equally

Key words: TYR-related protein 1, uveal melanoma, differential expression, immunocytochemistry, immunofluorescence every million individuals $(1,2)$. Due to its drug resistance and the high likelihood of metastasis to other organs, UM has a poor prognosis (3). Despite successful treatment of the primary tumor, nearly $40 \%$ of patients succumb to metastatic disease (4). In the clinic, the conventional treatments for UM are chemotherapy, radiotherapy and surgical excision, however, the efficacy of these treatments is limited. To overcome this problem, extensive studies have been performed with regard to immunotherapy, which have shown that this therapy is a significant constituent of the treatment for malignant melanomas $(5,6)$.

Tyrosinase (TYR) is a copper-containing enzyme and a type I membrane protein that is involved in the generation of melanin, the main pigment in vertebrates. TYR is encoded at the Tyr locus (formerly the albino or C locus). The protein catalyzes the initial conversion of tyrosine to 3,4-dihydroxyphenylalanine (DOPA), and may also catalyze the oxidation of DOPA to DOPA quinone and 5,6-dihydroxyindole to indole-5,6-quinone $(7,8)$. TYR is able to catalyze the oxylation of tyrosine to DOPA quinone directly (9-12). The TYR-related protein 1 (TYRP1) locus encodes a 75-kDa glycoprotein that exhibits an amino acid sequence homology to TYR of $43 \%$ (13). Furthermore, TYRP1 has been proposed to be a second, less efficient tyrosine hydroxylase (14), catalyzing the generation of DOPA from tyrosine. TYRP1 is believed to primarily stabilize and maintain the protein levels of TYR in humans $(15,16)$. As a marker, TYRP1 plays a crucial role in the immunotherapy of melanoma, and it has been widely studied for a number of years (17-19). Nonetheless, the complete mechanisms of TYRP1 activity have yet to be illuminated.

The present study examined the expression of TYRP1 in four human UM cell lines and one retinal pigment epithelium cell line at the mRNA, protein and morphological levels. Differential expression of TYRP1 by these UM cells was observed, providing novel insights with regard to TYRP1, which may be significant in further research and may be crucial for the development of treatments for UM.

\section{Materials and methods}

Cell lines and culture. The human UM cell lines, SP6.5, OM431, OCM1 and OCM290, were kindly provided by Professor John F. Marshall (Tumor Biology Laboratory, Cancer Research UK Clinical Center, John Vane Science Centre, 
London, UK) (20). The human retinal pigment epithelium (RPE) cell line was generously provided by the Department of Ophthalmology, Ruijin Hospital, Shanghai Jiao Tong University School of Medicine (Shanghai, China). The UM and RPE cells were cultured in Dulbecco's modified Eagle's medium (Gibco; Thermo Fisher Scientific, Inc., Waltham, MA, USA). All culture media were supplemented with $10 \%$ fetal bovine serum, and all cells were incubated at $37^{\circ} \mathrm{C}$ in a humidified incubator with $5 \% \mathrm{CO}_{2}$. The study was approved by the Animal Care and Use Committee at Shanghai Jiaotong University School of Medicine (Shanghai, China).

Reverse transcription-polymerase chain reaction (RT-PCR) analysis. Total cellular RNA was extracted using the TRIzol ${ }^{\mathbb{B}}$ Plus RNA Purification System (Gibco; Thermo Fisher Scientific, Inc.) according to the manufacturer's protocols. The RNA were reverse-transcribed using the Prime-Script 1st Strand cDNA Synthesis kit (Takara Bio, Inc., Otsu, Japan) in a $20 \mu \mathrm{l}$ volume with $1 \mu \mathrm{l}$ reverse transcriptase (MBI Fermentas, Inc.; Thermo Fisher Scientific, Inc.) according to the manufacturer's protocol. The PCR reaction system $(20 \mu \mathrm{l})$ contained $1 \mu \mathrm{g}$ cDNA, $1 \mu \mathrm{M}$ of each primer and $10 \mu \mathrm{l}$ Ex Taq solution (SYBR Premix Ex Taq $^{\mathrm{TM}}$ II kit; Takara Bio, Inc.). RT-PCR was performed on the ABI 9700 PCR machine (Applied Biosystems; Thermo Fisher Scientific, Inc.). RT-PCR was performed under the following conditions: $95^{\circ} \mathrm{C}$ for $10 \mathrm{~min}$ for 1 cycle, followed by 40 cycles of denaturation at $94^{\circ} \mathrm{C}$ for $30 \mathrm{sec}$, annealing at $60^{\circ} \mathrm{C}$ for $30 \mathrm{sec}$ and extension at $72^{\circ} \mathrm{C}$ for $30 \mathrm{sec}$. Premier Primer 5 software (Premier Biosoft, Palo Alto, CA, USA) was used to assess the PCR primers. The PCR primers were as follows: TYRP1 sense, 5'-GTAACAGCACCGAGGATGG-3' and antisense, 5'-TCC AAGCACTGAGCGACAT-3'; and glyceraldehyde-3-phosphate dehydrogenase (GAPDH) sense, 5'-TGGGGAAGGTGAAGG TCGG-3' and antisense, 5'-CTGGAAGATGGTGATGGG-3'. GAPDH was used as the internal control in PCR amplification. After staining with ethidium bromide and visualization under ultraviolet light, the amplified products were analyzed by electrophoresis on $2 \%$ agarose gels.

Quantitative (q)PCR. qPCR was performed using the aforementioned PCR program, with SYBR Premix Ex Taq II (Takara Bio, Inc.), on a Rotor-Gene 3000 Real-Time Thermo Cycler (Corbett Research, New South Wales, Australia) following the manufacturer's protocols (Takara Bio, Inc.), and the data were standardized against the quantification cycle of the GAPDH control. The extension steps were manipulated as follows: $95^{\circ} \mathrm{C}$ for $30 \mathrm{sec}$ for 1 cycle, followed by $95^{\circ} \mathrm{C}$ for $5 \mathrm{sec}$, $60^{\circ} \mathrm{C}$ for $30 \mathrm{sec}$ and $72^{\circ} \mathrm{C}$ for $15 \mathrm{sec}$ for 40 cycles.

Western blot analysis. As previously described (21), western blot analysis was performed to examine TYRP1 protein expression in the UM and RPE cells. Firstly, the cells were harvested, washed in cold phosphate-buffered saline (PBS) and then lysed with lysis buffer. Using the Bicinchoninic Acid Protein assay kit (Thermo Fisher Scientific Inc.), the protein samples were divided equally. In total, $30 \mathrm{mg}$ of protein extracts were generated and immunoblotting was performed according to standard protocols. Cell lysates were electrophoresed on $10 \%$ polyacrylamide slab gels and transferred to a nitrocellulose membrane. The membrane was incubated with the TYRP1
A

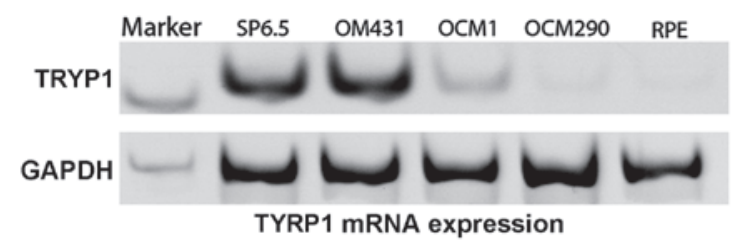

B

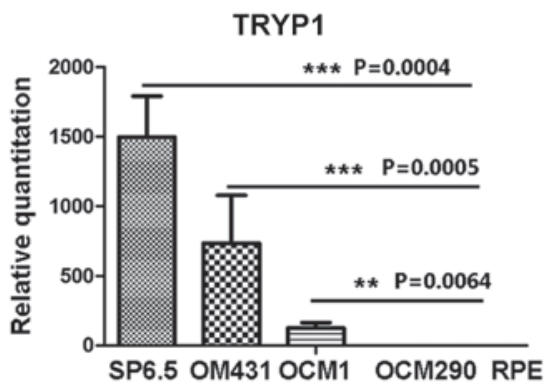

Figure 1. TYRP1 mRNA expression in human UM cells. (A) TYRP1 mRNA expression in the four UM cell lines and the RPE cells was determined by reverse transcription-PCR. (B) The relative quantitative analysis of TYRP1 mRNA expression in the 4 UM cell lines and the RPE cells was performed using quantitative PCR. The data represents three independent experiments and GAPDH was used as the control. TYRP1, tyrosinase-related protein 1; UM, uveal melanoma; RPE, retinal pigment epithelium; PCR, polymerase chain reaction; GAPDH, glyceraldehyde-3-phosphate dehydrogenase.

(mouse anti-human monoclonal; dilution, 1:1,000; Santa Cruz Biotechnology, Inc., Dallas, TX, USA) antibodies. An Odyssey Infrared Imaging System (LI-COR Biosciences, Lincoln, NE, USA) was used to visualize the signals. Monoclonal mouse TYRP1 (rabbit polyclonal; 1:1,000; Santa Cruz Biotechnology, Inc.) and primary monoclonal mouse anti- $\beta$-actin $(1: 5,000$; Sigma-Aldrich, St. Louis, MO, USA) were used as antibodies.

Immunocytochemical analysis. The collected cells were attached to glass slides, the smears of which were then fixed by $4 \%$ paraformaldehyde for 30 min and incubated with $0.1 \%$ Triton $\mathrm{X}-100$ and 5\% dimethylsulfoxide in PBS for $30 \mathrm{~min}$. Subsequent to being washed 3 times with cold PBS, the cells were subsequently blocked with $3 \%$ bovine serum albumin (BSA; Sigma-Aldrich) at $37^{\circ} \mathrm{C}$ for $30 \mathrm{~min}$. The cells were incubated with TYRP1 (rabbit anti-human polyclonal; dilution, 1:1,000; catalog no., sc-25543; Santa Cruz Biotechnology, Inc.,) antibody overnight at $4{ }^{\circ} \mathrm{C}$, and then subjected to the secondary antibody (goat anti-rabbit polyclonal; dilution, 1:500; catalog no., A0545; Sigma-Aldrich) for $30 \mathrm{~min}$. Following another wash in PBS for $15 \mathrm{~min}$, the cells were stained with 3,3'-diaminobenzidine (Dako, Carpinteria, CA, USA). Nuclear counterstaining with Harris stain was then performed for 3 min.

Immunofluorescence staining. The cells were prepared as aforementioned, and then incubated overnight with rabbit polyclonal TYRP1 antibody at $4^{\circ} \mathrm{C}$. Subsequently, the cells were gently rinsed 3 times for 15 min each time in cold PBS, followed by incubation with goat anti-rabbit immunoglobulin G secondary antibody (1:300 dilution in PBS/5\% BSA; Invitrogen; Thermo Fisher Scientific, Inc.) and 4',6-diamidino-2-phenylindole (1:1,000 dilution in PBS/5\% BSA) for $10 \mathrm{~min}$ at $37^{\circ} \mathrm{C}$ in the dark. Finally the cell smears were placed on coverslips and images were captured under a fluorescence microscope at 490-520 nm. 
A

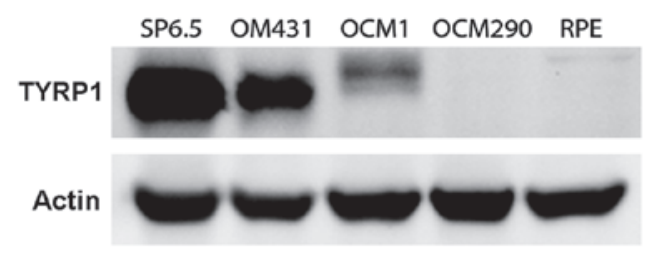

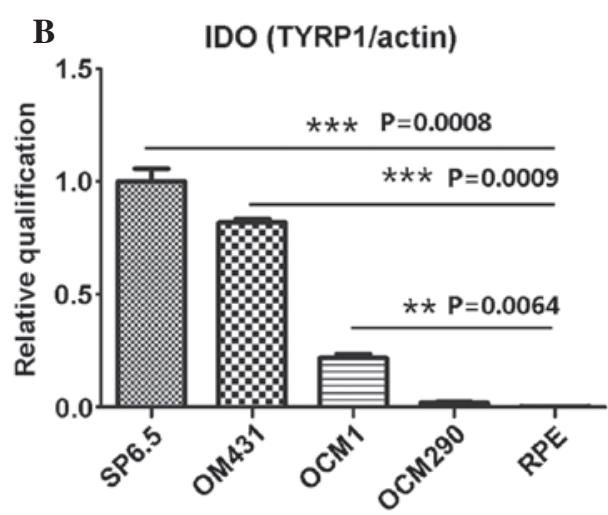

Figure 2. TYRP1 protein expression in human UM cells. (A) Western blot analysis of TYRP1 protein expression was performed using the TYRP1 antibody in RPE cells and four UM cell lines. (B) The protein bands were analyzed by a gradation scan, and the relative value was obtained from TYRP1/actin. IOD, integrated optical density. TYRP1, tyrosinase-related protein 1; UM, uveal melanoma; RPE, retinal pigment epithelium.

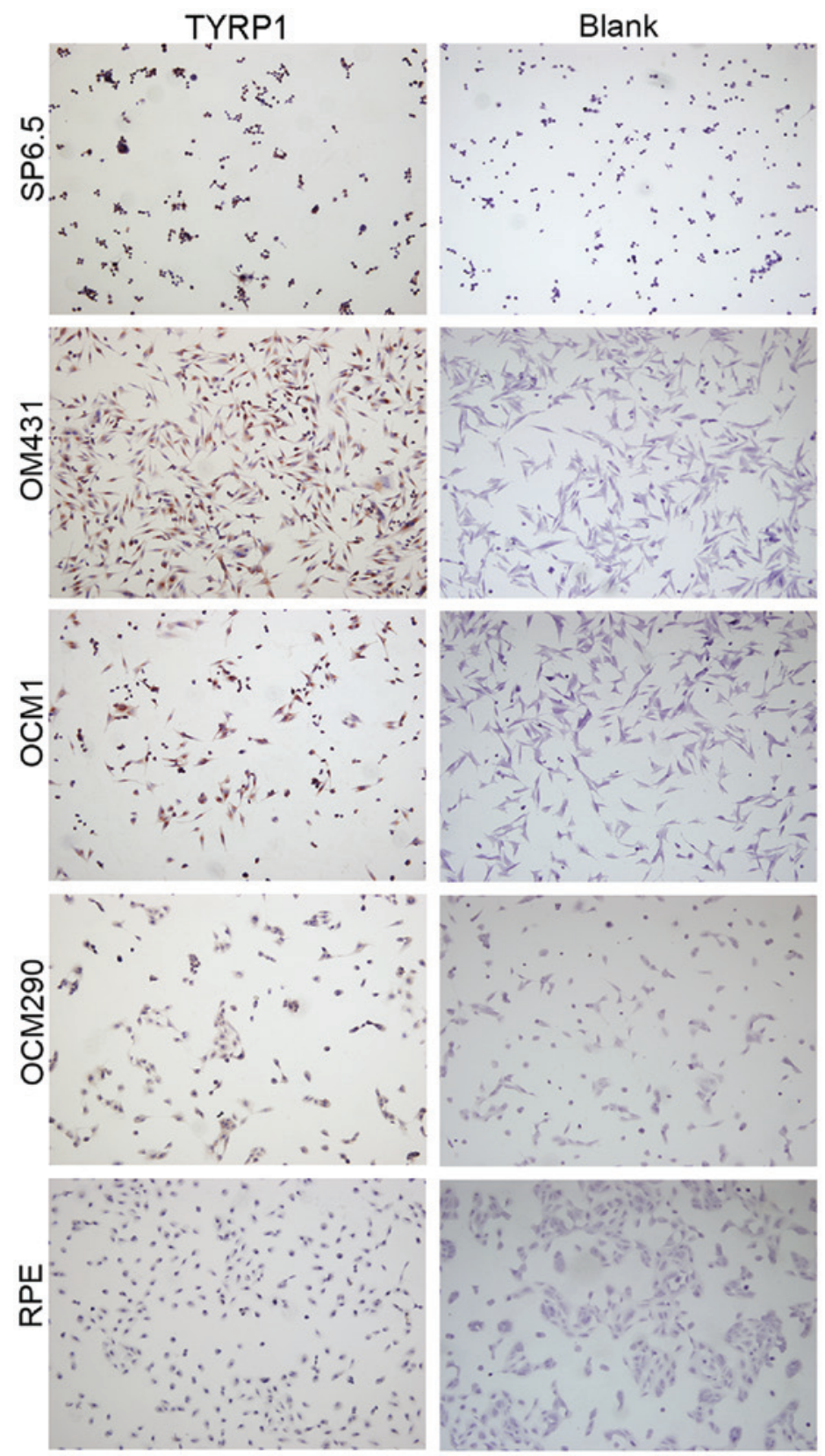

Figure 3. Immunohistochemical staining of TYRP1 in four UM cell lines and RPE cells. Nuclei were counterstained with Harris (blue), and TYRP1 was visualized with diaminobenzidine (brown). Phosphate-buffered saline was used instead of TYRP1 in the blank control group. Original magnification, x10. TYRP1, tyrosinase-related protein 1; UM, uveal melanoma; RPE, retinal pigment epithelium. 

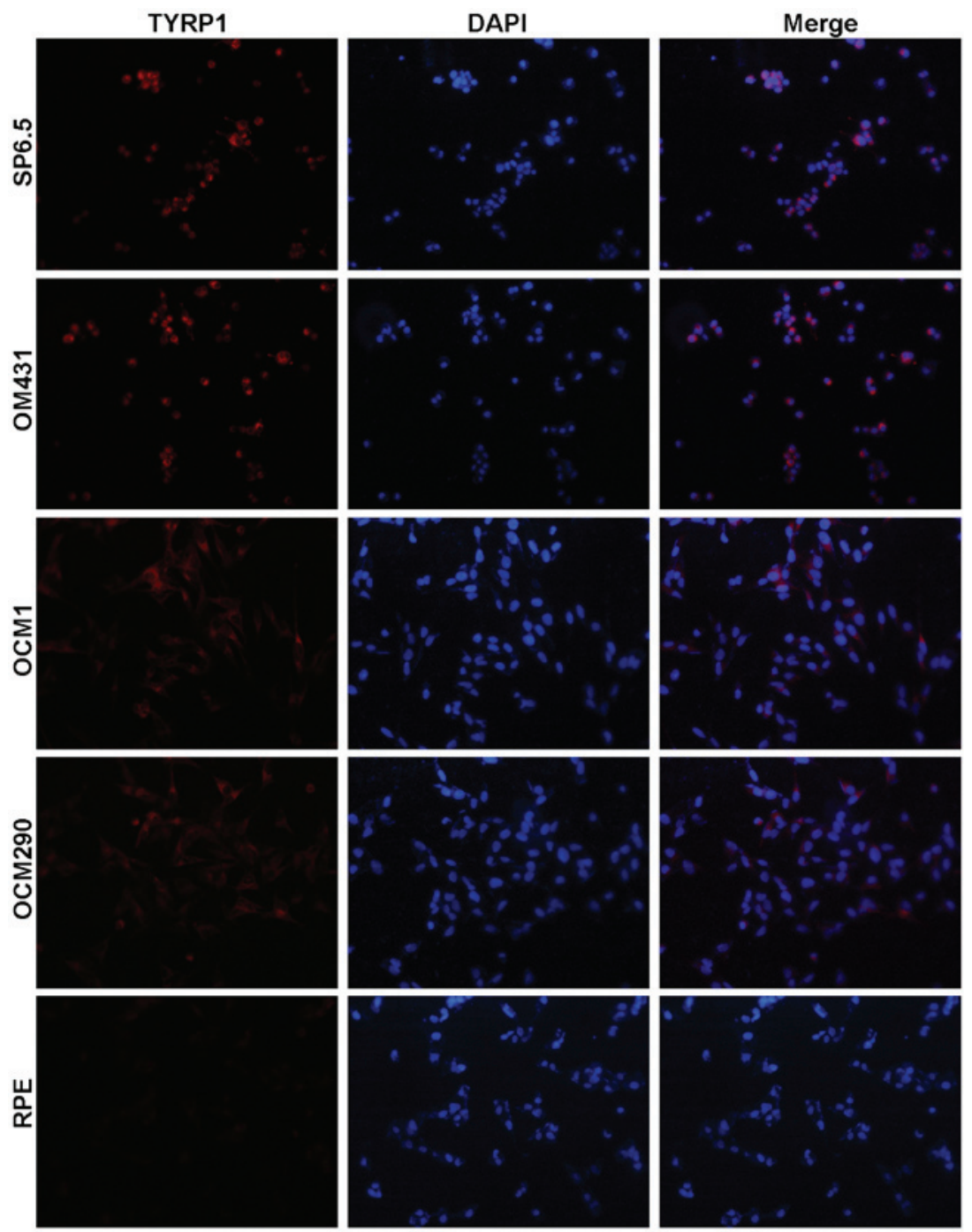

Figure 4. Immunofluorescence detection of TYRP1 in four uveal melanoma cell lines and RPE cells. Nuclei were stained with DAPI (blue), and TYRP1 was visualized with goat anti-rabbit immunoglobulin G secondary antibody (red). Original magnification, x20. TYRP1, tyrosinase-related protein 1; RPE, retinal pigment epithelium; DAPI, 4',6-diamidino-2-phenylindole.

Statistical analysis. Data are presented as the mean \pm standard deviation of three independent experiments. Statistical significance was assessed using the Student's two-tailed t-test and all statistical analyses were performed using SPSS 19.0 software (SPSS Inc., Chicago, IL, USA). P $<0.05$ was considered to indicate a statistically significant difference.

\section{Results}

Expression of TYRPI in UM cells at the mRNA level. The present study first investigated the mRNA expression of TYRP1. Experiments were performed on four human UM cell lines (SP6.5, OM431, OCM1 and OCM290), using the human retinal pigment epithelium cell line, RPE, as the control. The RT-PCR results clearly showed that the control RPE cells did not express TYRP1, while the UM cells expressed TYRP1 mRNA, with the exception of OCM290 cells ( $\mathrm{P}<0.01)$. SP6.5 cells expressed the highest level of TYRP1 mRNA, while OCM1 and OM431 cells produced less in comparison (Fig. 1A). qPCR was performed to confirm the differences in the expression of TYRP1 mRNA in these cell lines. The results were consistent with those of the
RT-PCR, and showed that the SP6.5 cells expressed the highest level of TYRP1 mRNA compared with the OM431 and OCM1 cells $(\mathrm{P}=0.015$ and $\mathrm{P}=0.019$, respectively).

Expression of TYRP1 in UM cells at the protein level. TYRP1 protein expression in the UM and RPE cells was determined by western blot analysis. The results were consistent with those of the PCR, and showed that the SP6.5 cells strongly expressed TYRP1, while the OCM1 and OM431 cells expressed relatively lower protein levels $(\mathrm{P}<0.01)$. In the RPE and OCM290 cells, TYRP1 protein expression was not found (Fig. 2).

Expression of TYRPI in UM cells at the cellular level. Immunocytochemistry was used to determine the expression of TYRP1 in the UM and RPE cells. In comparison to the blank control group, the SP6.5, OM431 and OCM1 cells were markedly stained $(\mathrm{P}=0.005, \mathrm{P}=0.008$ and $\mathrm{P}=0.032$, respectively) (Fig. 3), while no staining was observed in the OCM290 and RPE cells. Moreover, positive staining was found in the OCM1 and OM431 cells. The presence of TYRP1 protein expression in these cells was confirmed by immunofluorescence staining (Fig. 4). 


\section{Discussion}

UM exhibits the highest incidence among adult primary intraocular tumors $(1,2)$. Treatment strategy design for UM is associated with the two main issues of drug resistance and a high metastatic rate. With the development of gene research, gene therapy and immunotherapy have become more important and their utility has also been improved. Immunotherapy has been extensively studied, and is currently a significant feature of the treatment regimen for malignant melanomas $(5,6)$. Extensive studies have been performed on UM, including studies for a series of other proteins besides TYRP1.

In melanocytes, TYRP1 is significantly expressed and has been identified during melanin synthesis in the melanosome. TYRP1 is specifically expressed in melanocytes and is involved in melanin synthesis within melanosomes, as are the other members of the tyrosinase related protein family, which includes tyrosinase (TYR), and dopachrome tautomerase (TYRP2). RPE cells in human adults do not generate melanin in vitro under ordinary culture conditions (22-26). Smith-Thomas et al reported that TYRP1 and TYRP2 protein was not detected in cultured human RPE cells, however, this study only used a non-quantitative immunostaining method (23).

In the present study, it was demonstrated that the expression of TYRP1 was markedly different in four UM cell lines. Thus, the effects of immunotherapy mediated by the antigenicity of TYRP1 in patients with UM can differ. These findings can clearly lead to distinctly varied prognoses. The expression of TYRP1 in the present study was as follows: TYRP1 mRNA expression in the OCM431 and OCM1 cells occurred at similar levels, while expression was slightly lower in the SP6.5 cells, and almost no TYPR1 mRNA was expressed in the OCM290 cells. The TYRP1 protein level corresponded with the TYRP1 mRNA level. These findings represent the initial stages of understanding TYRP1 expression in UM cells. The functional significance and regulatory mechanisms of TYRP1 have yet to be defined, however, findings such as aforementioned may result in the development of immunotherapy for UM

\section{Acknowledgements}

This study was supported by the Scientific Research Program of National Health and Family Planning Commission of China (grant no. 201402014), The National Natural Science Foundation of China (grant nos. 81372469 and 81372909), and The Science and Technology Commission of Shanghai (grant nos. 13JC14006202, 12ZR1417300 and 13ZR1423600).

\section{References}

1. Stang A and Jöckel KH: Trends in the incidence of ocular melanoma in the United States, 1974-1998. Cancer Causes Control 15: 95-96, 2004.

2. Singh AD, Bergman L and Seregard S: Uveal melanoma: Epidemiologic aspects. Ophthalmol Clin North Am 18: 75-84, 2005.

3. Flaherty LE, Unger JM, Liu PY, Mertens WC and Sondak VK: Metastatic melanoma from intraocular primary tumors: The southwest oncology group experience in phase II advanced melanoma clinical trials. Am J Clin Oncol 21: 568-572, 1998.

4. Kujala E, Tuomaala S, Eskelin S and Kivelä T: Mortality after uveal and conjunctival melanoma: Which tumour is more deadly? Acta Ophthalmol 87: 149-153, 2009.
5. Alexandrescu DT, Ichim TE, Riordan NH, Marincola FM, Di Nardo A, Kabigting FD and Dasanu CA: Immunotherapy for melanoma: Current status and perspectives. J Immunother 33: 570-590, 2010.

6. Jandus C, Speiser D and Romero P: Recent advances and hurdles in melanoma immunotherapy. Pigment Cell Melanoma Res 22: 711-723, 2009.

7. Tripathi RK, Hearing VJ, Urabe K, Aroca P and Spritz RA: Mutational mapping of the catalytic activities of human tyrosinase. J Biol Chem 267: 23707-23712, 1992.

8. Korner A and Pawelek J: Mammalian tyrosinase catalyzes three reactions in the biosynthesis of melanin. Science 217: 1163-1165, 1982.

9. Cooksey CJ, Garratt PJ, Land EJ, Pavel S, Ramsden CA, Riley PA and Smit NP: Evidence of the indirect formation of the catecholic intermediate substrate responsible for the autoactivation kinetics of tyrosinase. J Biol Chem 272: 26226-26235, 1997.

10. Hearing VJ and Jiménez M: Mammalian tyrosinase-the critical regulatory control point in melanocyte pigmentation. Int J Biochem 19: 1141-1147, 1987.

11. Jiménez M, Maloy WL and Hearing VJ: Specific identification of an authentic clone for mammalian tyrosinase. J Biol Chem 264: 3397-3403, 1989

12. Jimenez M, Tsukamoto $\mathrm{K}$ and Hearing VJ: Tyrosinases from two different loci are expressed by normal and by transformed melanocytes. J Biol Chem 266: 1147-1156, 1991.

13. Jackson IJ: A cDNA encoding tyrosinase-related protein maps to the brown locus in mouse. Proc Natl Acad Sci USA 85: 4392-4396, 1988

14. Boissy RE, Zhao H, Oetting WS, Austin LM, Wildenberg SC, Boissy YL, Zhao Y, Sturm RA, Hearing VJ, King RA and Nordlund JJ: Mutation in and lack of expression of tyrosinase-related protein-1 (TRP-1) in melanocytes from an individual with brown oculocutaneous albinism: A new subtype of albinism classified as 'OCA3'. Am J Hum Genet 58: 1145-1156, 1996.

15. Kobayashi T, Imokawa G, Bennett DC and Hearing VJ: Tyrosinase stabilization by TRYP1 (the brown locus protein). J Biol Chem 273: 31801-31805, 1998.

16. Manga P, Sato K, Ye L, Beermann F, Lamoreux ML and Orlow SJ: Mutational analysis of the modulation of tyrosinase by tyrosinase-related proteins 1 and 2 in vitro. Pigment Cell Res 13: 364-374, 2000

17. de Vries TJ, Trancikova D, Ruiter DJ and van Muijen GN: High expression of immunotherapy candidate proteins gp100, MART-1, tyrosinase and TRP-1 in uveal melanoma. $\mathrm{Br}$ J Cancer 78: 1156-1161, 1998.

18. Shidham VB, Qi D, Rao RN, Acker SM, Chang CC, Kampalath B, Dawson G, Machhi JK and Komorowski RA: Improved immunohistochemical evaluation of micrometastases in sentinel lymph nodes of cutaneous melanoma with 'MCW melanoma cocktail'-a mixture of monoclonal antibodies to MART-1, Melan-A, and tyrosinase. BMC Cancer 3: 15, 2003.

19. Kawakami Y, Robbins PF, Wang RF, Parkhurst M, Kang X and Rosenberg SA: The use of melanosomal proteins in the immunotherapy of melanoma. J Immunother 21: 237-246, 1998.

20. Jia R, Jiao Z, Xu X, Wang J, Zhou Y, Song X, Ge S and Fan X: Functional significance of B7-H1 expressed by human uveal melanoma cells. Mol Med Rep 4: 163-167, 2011.

21. Song X, Zhou Y, Jia R, Xu X, Wang H, Hu J, Ge S and Fan X: Inhibition of retinoblastoma in vitro and in vivo with conditionally replicating oncolytic adenovirus H101. Invest Ophthalmol Vis Sci 51: 2626-2635, 2010.

22. Boulton $\mathrm{M}$ and Dayhaw-Barker P: The role of the retinal pigment epithelium: Topographical variation and ageing changes. Eye (Lond) 15: 384-389, 2001.

23. Smith-Thomas L, Richardson P, Thody AJ, Graham A, Palmer I, Flemming L, Parsons MA, Rennie IG and MacNeil S: Human ocular melanocytes and retinal pigment epithelial cells differ in their melanogenic properties in vivo and in vitro. Curr Eye Res 15: 1079-1091, 1996.

24. Flood MT, Gouras P and Kjeldbye H: Growth characteristics and ultrastructure of human retinal pigment epithelium in vitro. Invest Ophthalmol Vis Sci 19: 1309-1320, 1980.

25. Newsome DA: Retinal pigmented epithelium culture: Current applications. Trans Ophthalmol Soc UK 103: 458-466, 1983.

26. Albert DM, Ruzzo MA, McLaughlin MA, Robinson NL, Craft JL and Epstein J: Establishment of cell lines of uveal melanoma. Methodology and characteristics. Invest Ophthalmol Vis Sci 25: 1284-1299, 1984 